\title{
An Application of Sombor Index over a Special Class of Semigroup Graph
}

\author{
Seda Oğuz Ünal \\ Department of Secondary School Science and Mathematics Education, Education Faculty, Cumhuriyet University, \\ Sivas 58140, Turkey \\ Correspondence should be addressed to Seda Oğuz Ünal; sdaoguz@gmail.com
}

Received 18 August 2021; Revised 18 October 2021; Accepted 25 October 2021; Published 15 November 2021

Academic Editor: R. Vadivel

Copyright (c) 2021 Seda Oğuz Ünal. This is an open access article distributed under the Creative Commons Attribution License, which permits unrestricted use, distribution, and reproduction in any medium, provided the original work is properly cited.

Recently, Gutman introduced a class of novel topological invariants named Sombor index which is defined $\operatorname{as} S O(G)=\sum_{u v \in E(G)} \sqrt{\left(d_{u}\right)^{2}+\left(d_{v}\right)^{2}}$. In this study, the Sombor index of monogenic semigroup graphs, which is an important class of algebraic structures, is calculated.

\section{Introduction and Preliminaries}

The monogenic semigroup graph is inspired by zero divisor graphs. Therefore, before moving on to the main topic, we will focus on the studies on zero divisor graphs (see [1-4]). In relation to the study of zero divisor graphs that has many authors researching commutative and noncommutative rings and how it has advanced, DeMeyer et al. [5, 6] have developed research on commutative and noncommutative semigroups related to zero divisor graphs. The authors in [7] utilised the adjacent rule of vertices while still keeping the original idea. The authors determined a finite multiplicative monogenic semigroup with 0 as follows:

$$
S_{M}=\left\{0, x, x^{2}, x^{3}, \ldots, x^{n}\right\} .
$$

By utilizing the idea defined in $[5,6]$, the authors obtained a new graph related to monogenic semigroups in [7]. The vertices of this graph are all nonzero elements in $S_{M}$ and for any two different vertices $x^{i}$ and $x^{j}$ where $(1 \leq i, j \leq n)$ are linked to each other, if and only if $i+j>n$. There are many studies concerning monogenic semigroup graphs which were published by Akgüneş et al. (see for example [8-10]).

In chemistry, topological indices have been around for more than half a century [11]. In newer times, they are being extensively investigated also by mathematicians. These indices are used to model structural properties of molecules and provide information of value for physical chemistry, material science, pharmacology, environmental sciences, and biology [12]. Recently, a new such graph-based topological index, called Sombor index, was put forward by Gutman [13]. Initially, the index was applied in chemistry [14-18] and soon attracted the interest of mathematicians [19-22]. Eventually, however, the Sombor index found applications also in network science and was used for modeling dynamical effects in biology, social, and technological complex systems [23]. It seems that this index became interesting also for military purposes [24]. All this happened within less than one year since the publication of the paper [13]. In view of this wide research activity on Sombor index, it may be of interest to seek for its deeper algebraic connections. In this paper, we report some results relating the Sombor index with an important class of algebraic structures, namely, with monogenic semigroups.

For a graph $G$, its edge set and vertex set are denoted by $E(G)$ and $V(G)$, respectively.

Sombor index discovered by; Gutman [13] is one of the vertex-degree-based topological indices defined by

$$
S O(G)=\sum_{u v \in E(G)} \sqrt{\left(d_{u}\right)^{2}+\left(d_{v}\right)^{2}},
$$


because the function $F(x, y)=\sqrt{x^{2}+y^{2}}$ was not utilised.

Also, as a reminder, for a real number $r$, we identify by $\lfloor r\rfloor$ the greatest integer $\leq r$, and by $\lceil r\rceil$, the least integer $\geq r$. It is clear that $r-1<\lfloor r\rfloor \leq r$ and $r \leq\lceil r\rceil<r+1$. However, for a natural number $n$, we have

$$
\left\lfloor\frac{n}{2}\right\rfloor= \begin{cases}\frac{n}{2}, & \text { if } n \text { is even, } \\ \frac{n-1}{2}, & \text { if } n \text { is odd. }\end{cases}
$$

In this paper, we focus on determining the explicit formula of Sombor index of the monogenic semigroup graph.

\section{An Algorithm}

The authors in [8] to simplify their research gave the algorithm concerning the neighborhood of vertices by utilizing the initial statement of monogenic semigroup graph. We will use this algorithm in our main theorem in the next section.

$I_{n}$ : the vertex $x^{n}$ is adjoining to every vertex $x^{i_{1}}\left(1 \leq i_{1} \leq n-1\right)$ except itself.

$I_{n-1}$ : the vertex $x^{n-1}$ is adjoining to every vertex $x^{i_{2}}\left(2 \leq i_{2} \leq n-2\right)$ except itself and the vertex $x^{n}$.

$I_{n-2}$ : the vertex $x^{n-2}$ is adjoining to every vertex $x^{i_{3}}\left(3 \leq i_{3} \leq n-3\right)$ except itself and the vertices $x^{n}$ and $x^{n-1}$.
Carrying on the algorithm this way, we get the following result, depending on whether the number $n$ is odd or even.

If $n$ is even,

$I_{(n / 2)+2}$ : the vertex $x^{(n / 2)+2}$ is adjoining not only to the vertices $x^{(n / 2)-1}, x^{(n / 2)}$, and $x^{(n / 2)+1}$ but also to the vertices $x^{n}, x^{n-1}, x^{n-2}, \ldots, x^{(n / 2)+3}$.

$I_{(n / 2)+1}$ : the vertex $x_{(n / 2)+1}$ is adjoining not only to the single vertex $x^{(n / 2)}$ but also to the vertices $x^{n}, x^{n-1}$, $x^{n-2}, \ldots, x^{(n / 2)+2}$

If $n$ is odd,

$I_{(n+1) / 2}$ : the vertex $x^{(n+1) / 2+2}$ is adjoining not only to the vertices $x^{(n+1 / 2)-2}, x^{(n+1 / 2)-1}, x^{(n+1 / 2)}$, and $x^{(n+1 / 2)+1}$ also adjoining to the vertices $x^{n}, x^{n-1}, x^{n-2}, \ldots, x^{(n+1 / 2)+3}$. $I^{(n+1) / 2+1}$ : the vertex $x^{(n+1) / 2+1}$ is adjoining not only to the vertices $x^{(n+1 / 2)-1}$ and $x^{(n+1 / 2)}$ also adjoining to the vertices $x^{n-1}, x^{n-2}, \ldots, x^{(n+1) / 2+2}$.

In the lemma given below, the degrees of vertices $x^{1}, x^{2}, \ldots, x^{n} \in \Gamma\left(S_{M}\right)$ are denoted by $d_{1}, d_{2}, \ldots, d_{n}$. There are many studies on the degree series. Regarding this, you can refer to $[7,25]$ and references cited in these studies. In fact, in the lemma below, it is mentioned that there is an ordering between the degrees $d_{1}, d_{2}, \ldots, d_{n}$. You can reach the proof of this lemma from [7], as well as from the algorithm given above (see [8]).

Lemma 1.

$$
d_{1}=1, d_{2}=2, \ldots, d_{\lfloor n / 2\rfloor}=\left\lfloor\frac{n}{2}\right\rfloor, d_{\lfloor n / 2\rfloor+1}=\left\lfloor\frac{n}{2}\right\rfloor, d_{\lfloor n / 2\rfloor+2}=\left\lfloor\frac{n}{2}\right\rfloor+1, \ldots, d_{n}=n-1
$$

Remark 1. Paying attention to Lemma 1, the repeated terms are given in the following:

$$
d_{\lfloor n / 2\rfloor}=\left\lfloor\frac{n}{2}\right\rfloor=d_{\lfloor n / 2\rfloor+1}
$$

Therefore, the degree of $d_{n}$ is denoted by $n-1$, although the number of vertices is $n$.

\section{Calculating Sombor Index of $\Gamma\left(S_{M}\right)$}

In this section, we will obtain an exact formula of Sombor index over monogenic semigroup graph.

Theorem 1. For any monogenic semigroup $S_{M}$ as given in (1), the Sombor index of the graph $\Gamma\left(S_{M}\right)$ is

$$
S O\left(\Gamma\left(S_{M}\right)\right)= \begin{cases}\sum_{k=1}^{(n / 2)-1} \sum_{i=k}^{n-k-1} \sqrt{(n-k)^{2}+i^{2}}+\sum_{k=1}^{n / 2} \sqrt{(n-k)^{2}+\left(\frac{n}{2}\right)^{2}}, & \text { if } n \text { is even, } \\ \sum_{k=1}^{(n-1) / 2} \sum_{i=k}^{n-k-1} \sqrt{(n-k)^{2}+i^{2}}+\sum_{k=1}^{(n-1) / 2} \sqrt{(n-k)^{2}+\left(\frac{n}{2}\right)^{2}}, & \text { if } n \text { is odd. }\end{cases}
$$

Proof. Since our aim is to formulate $S O\left(\Gamma\left(S_{M}\right)\right)$ in terms of the total number of degrees, we need to treat the sum as the sum of different blocks and then calculate each separately. During our calculations, we will use the algorithm given in 
Section 2 here, as it offers a very systematic way of calculating the degrees of vertices. We will also make use of equations (3) and (4) and Remark 1
If $n$ is odd,

$$
\begin{aligned}
& {[S O]\left(\Gamma\left(S_{M}\right)=\sqrt{d_{n}^{2}+d_{1}^{2}}+\sqrt{d_{n}^{2}+d_{2}^{2}}+\sqrt{d_{n}^{2}+d_{3}^{2}}+\cdots+\sqrt{d_{n}^{2}+d_{n-2}^{2}}+\sqrt{d_{n}^{2}+d_{n-1}^{2}}+\sqrt{d_{n-1}^{2}+d_{2}^{2}}+\sqrt{d_{n-1}^{2}+d_{3}^{2}}\right.} \\
& +\cdots+\sqrt{d_{n-1}^{2}+d_{n-2}^{2}}+\cdots+\sqrt{d_{(n+1 / 2)+2}^{2}+d_{(n+1 / 2)-2}^{2}}+\sqrt{d_{(n+1 / 2)+2}^{2}+d_{(n+1 / 2)-1}^{2}}+\sqrt{d_{(n+1 / 2)+2}^{2}+d_{(n+1 / 2)}^{2}} \\
& \quad+\sqrt{d_{(n+1 / 2)+2}^{2}+d_{(n+1 / 2)+1}^{2}}+\sqrt{d_{(n+1 / 2)+1}^{2}+d_{(n+1 / 2)-1}^{2}}+\sqrt{d_{(n+1 / 2)+1}^{2}+d_{(n+1 / 2)}^{2}} .
\end{aligned}
$$

As a result, the Sombor index of $\Gamma\left(S_{M}\right)$ is written as the sum below:

$$
[\mathrm{SO}]\left(\Gamma\left(S_{M}\right)=\sum_{i j \in E(G)} \sqrt{d_{i}^{2}+d_{j}^{2}}=[\mathrm{SO}]_{n}+[\mathrm{SO}]_{n-1}+\cdots+[\mathrm{SO}]_{(n+1 / 2)+2}+[\mathrm{SO}]_{(n+1 / 2)+1}\right.
$$

When calculating the Sombor index sum, we will write the smallest degree at the end of the line, so we will get a second total and this will provide us with ease of operation.
By the way, while making these calculations, we use the equation $\lfloor n / 2\rfloor=((n-1) /(2))$ given in (2) for the case where $n$ is odd.

$$
\begin{aligned}
{[S O]_{n}=} & \sqrt{(n-1)^{2}+1^{2}}+\sqrt{(n-1)^{2}+2^{2}}+\sqrt{(n-1)^{2}+3^{2}}+\cdots+\sqrt{(n-1)^{2}+\left\lfloor\frac{n}{2}\right\rfloor^{2}}+\cdots+ \\
& +\sqrt{(n-1)^{2}+(n-2)^{2}}+\sqrt{(n-1)^{2}+\left\lfloor\frac{n}{2}\right\rfloor^{2}} \\
= & \sum_{i=1}^{n-2} \sqrt{(n-i)^{2}+i^{2}}+\sqrt{(n-1)^{2}+\left(\frac{n-1}{2}\right)^{2}} .
\end{aligned}
$$

If similar operations applied in $[S O]_{n}$ are applied in $[\mathrm{SO}]_{n-1}$, we obtain

$$
\begin{gathered}
{[S O]_{n-1}=\sum_{i=2}^{n-3} \sqrt{(n-i)^{2}+i^{2}}+\sqrt{(n-2)^{2}+\left(\frac{n-1}{2}\right)^{2}}} \\
{[S O]_{(n+1) / 2+2}=\sqrt{\left(\frac{n+3}{2}\right)^{2}+\left(\frac{n-3}{2}\right)^{2}}+\sqrt{\left(\frac{n+3}{2}\right)^{2}+\left(\frac{n-1}{2}\right)^{2}}+\sqrt{\left(\frac{n+3}{2}\right)^{2}+\left(\frac{n-1}{2}\right)^{2}}+\sqrt{\left(\frac{n+3}{2}\right)^{2}+\left(\frac{n+1}{2}\right)^{2}}}
\end{gathered}
$$

and finally,

$$
[S O]_{(n+1) / 2+1}=\sqrt{\left(\frac{n+1}{2}\right)^{2}+\left(\frac{n-1}{2}\right)^{2}}+\sqrt{\left(\frac{n+1}{2}\right)^{2}+\left(\frac{n-1}{2}\right)^{2}}
$$




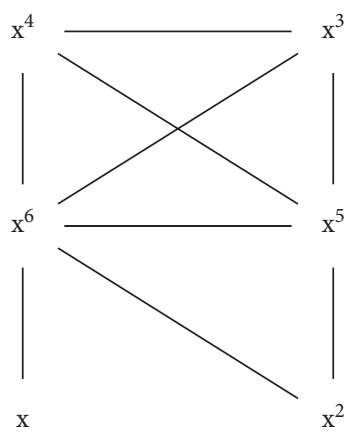

FIGURE 1: $S_{M}^{6}$ monogenic semigroup graph.

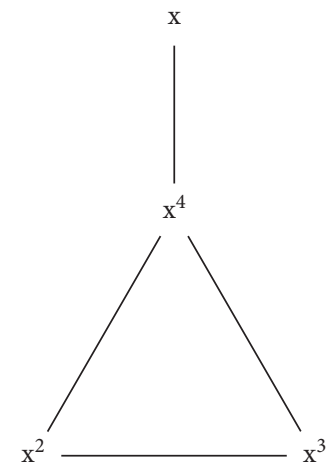

FIGURE 2: $S_{M}^{4}$ monogenic semigroup graph (corresponding hydrogen-suppressed molecular graph).

Hence,

$$
[S O]_{n}+[S O]_{n-1}+\cdots+[S O]_{(n+1) / 2+2}+[S O]_{(n+1) / 2+1}=\sum_{k=1}^{(n-1) / 2} \sum_{i=k}^{n-k-1} \sqrt{(n-k)^{2}+i^{2}}+\sum_{k=1}^{(n-1) / 2} \sqrt{(n-k)^{2}+\left(\frac{n}{2}\right)^{2}}
$$

If we follow similar steps as if $n$ is odd, we will get the following sum if $n$ is even:

$$
[S O]_{n}+[S O]_{n-1}+\cdots+[S O]_{(n / 2)+2}+[S O]_{(n / 2)+1}=\sum_{k=1}^{(n / 2)-1} \sum_{i=k}^{n-k-1} \sqrt{(n-k)^{2}+i^{2}}+\sum_{k=1}^{(n / 2)} \sqrt{(n-k)^{2}+\left(\frac{n}{2}\right)^{2}} .
$$

Corollary 1. In [26, 27], the authors exhibited that the Sombor index can be an integer in several graph structures. In monogenic semigroup graphs, it is seen that it is not possible for the Sombor index to take an integer value according to the formula given in Theorem 1.

We will give the following examples to reinforce Theorem 1 .
Example 1. Consider the monogenic semigroup $S_{M}^{6}$ given below and calculate the Sombor index of $\Gamma\left(S_{M}^{6}\right)$ graph by applying the rule given in Theorem 1 :

$$
S_{M}^{6}=\left\{x, x^{2}, x^{3}, x^{4}, x^{5}, x^{6}\right\} \cup\{0\} .
$$

Monogenic semigroup graphs, which are defined with inspiration from zero divisor graphs, also contain the 0 
element. Because the vertices of $x^{i}$ and $x^{j}$, which are taken arbitrarily in the monogenic semigroup, can be connected with each other, that is, the necessary and sufficient condition for the condition of $x^{i} x^{j}=0$ is to be $i+j>n$. In line with this information, the $S_{M}^{6}$ graph is given in Figure 1 .

$$
\begin{aligned}
S O\left(\Gamma\left(S_{M}^{6}\right)=\right. & \sum_{k=1}^{2} \sum_{i=k}^{5-k} \sqrt{(6-k)^{2}+i^{2}}+\sum_{k=1}^{3} \sqrt{(6-k)^{2}+(3)^{2}} \\
= & \sqrt{5^{2}+1^{2}}+\sqrt{5^{2}+2^{2}}+\sqrt{5^{2}+3^{2}}+\sqrt{5^{2}+4^{2}}+\sqrt{4^{2}+2^{2}} \\
& +\sqrt{4^{2}+3^{2}}+\sqrt{5^{2}+3^{2}}+\sqrt{4^{2}+3^{2}}+\sqrt{3^{2}+3^{2}}
\end{aligned}
$$

In the example below, the Sombor index of the corresponding hydrogen-suppressed molecular graph, which is equivalent to $\Gamma\left(S_{M}^{4}\right)$ monogenic semigroup graph, is calculated.
Example 2. The Sombor index of the monogenic semigroup $S_{M}^{4}$ given below is calculated by applying Theorem 1 .

$$
S_{M}^{4}=\left\{x, x^{2}, x^{3}, x^{4}\right\} \cup\{0\}
$$

The $S_{M}^{4}$ graph is given in Figure 2 .

$$
\begin{aligned}
S O\left(\Gamma\left(S_{M}^{4}\right)\right. & =\sum_{k=1} \sum_{i=1}^{2} \sqrt{(4-k)^{2}+i^{2}}+\sum_{k=1}^{2} \sqrt{(4-k)^{2}+(2)^{2}} \\
& =\sqrt{3^{2}+1^{2}}+\sqrt{3^{2}+2^{2}}+\sqrt{3^{2}+2^{2}}+\sqrt{2^{2}+2^{2}}
\end{aligned}
$$

As can be seen, the Sombor index of a monogenic semigroup graph can be calculated very easily with the given formula in Theorem 1.

\section{Data Availability}

No data were used to support this study.

\section{Conflicts of Interest}

The author declares no conflicts of interest.

\section{Acknowledgments}

The author wishes to express his appreciation to Professor Dr. Ivan Gutman for his invaluable support and expertise during the research phase of this paper.

\section{References}

[1] D. D. Anderson and M. Naseer, "Beck's coloring of a commutative ring," Journal of Algebra, vol. 159, pp. 500-514, 1991.

[2] D. F. Anderson and P. S. Livingston, "The zero-divisor graph of a commutative ring," Journal of Algebra, vol. 217, no. 2, pp. 434-447, 1999.

[3] D. F. Anderson and A. Badawi, "On the zero-divisor graph of a ring," Communications in Algebra, vol. 36, no. 8, pp. 3073-3092, 2008.

[4] I. Beck, "Coloring of commutative rings," Journal of Algebra, vol. 116, no. 1, pp. 208-226, 1988.
[5] F. DeMeyer and L. DeMeyer, "Zero divisor graphs of semigroups," Journal of Algebra, vol. 283, no. 1, pp. 190-198, 2005.

[6] F. R. DeMeyer, T. McKenzie, and K. Schneider, "The zerodivisor graph of a commutative semigroup," Semigroup Forum, vol. 65, no. 2, pp. 206-214, 2002.

[7] K. C. Das, N. Akgüneş, and A. S. Çevik, "On a graph of monogenic semigroup," Journal of Inequalities and Applications, vol. 44, 2013.

[8] N. Akgüneş, K. C. Das, and A. S. Çevik, "Topological indices on a graph of monogenic semigroups in topics in chemical graph theory," in Mathematical Chemistry Monographs, I. Gutman, Ed., University of Kragujevac and Faculty of Science Kragujevac, Kragujevac, Serbia, 2014.

[9] N. Akgüneş and B. Çağan, "On the dot product of graphs over monogenic semigroups," Applied Mathematics and Computation, vol. 322, pp. 1-5, 2018.

[10] N. Akgüneş, "A further note on the graph of monogenic semigroups," Konuralp Journal of Mathematics, vol. 6, no. 1, pp. 49-53, 2018.

[11] R. Todeschini and V. Consonni, Molecular Descriptors for Chemoinformatics, Wiley VCH, Weinheim, Germany, 2009.

[12] J. Devillers and A. T. Balaban, Topological Indices and Related Descriptors in QSAR and QSPR, Gordon \& Breach, Amsterdam, Netherlands, 1999.

[13] I. Gutman, "Geometric approach to degree-based topological indices: sombor indices," MATCH Commun. Math. Comput. Chem.vol. 86, pp. 11-16, 2021.

[14] S. Alikhani and N. Ghanbari, "Sombor index of polymers," MATCH Communications in Mathematical and in Computer Chemistry, vol. 86, pp. 715-728, 2021.

[15] S. Amin, A. U. Rehman Virk, M. A. Rehman, and N. A. Shah, "Analysis of dendrimer generation by Sombor indices," 
Hindawi Journal of Chemistry, vol. 2021, Article ID 9930645, 11 pages, 2021.

[16] R. Cruz, I. Gutman, and J. Rada, "Sombor index of chemical graphs," Applied Mathematics and Computation, vol. 399, Article ID 126018, 2021.

[17] H. Liu, L. You, and Y. Huang, "Ordering chemical graphs by Sombor indices and its applications," MATCH Communications in Mathematical and in Computer Chemistry, vol. 87, 2022 In press.

[18] I. Redzepovic, "Chemical applicability of Sombor indices," Journal of the Serbian Chemical Society, vol. 86, no. 5, pp. 445-457, 2021.

[19] K. C. Das, A. S. Çevik, I. N. Cangül, and Y. Shang, "On Sombor index," Symmetry, vol. 13, p. 140, 2021.

[20] B. Horoldagva and C. Xu, "On Sombor index of graphs," MATCH Communications in Mathematical and in Computer Chemistry, vol. 86, pp. 793-713, 2021.

[21] I. Milovanovic, E. Milovanovic, A. Ali, and M. Matejic, "Some results on the Sombor indices of graphs," Contributions to Mathematics, vol. 3, pp. 59-67, 2021.

[22] J. Rada, J. M. Rodríguez, and J. M. Sigarreta, "General properties on Sombor indices," Discrete Applied Mathematics, vol. 299, pp. 87-97, 2021.

[23] Y. Shang, "Sombor index and degree-related properties of simplicial networks," Applied Mathematics and Computation, In press.

[24] I. Gutman, "Spectrum and energy of the Sombor matrix," Military Technical Courier, vol. 69, pp. 551-561, 2021.

[25] N. Akgüneş and A. S. Çevik, "A new bound of radius of irregularity index," Applied Mathematics and Computation, vol. 219, pp. 5750-5753, 2013.

[26] T. Réti, T. Došlic, and A. Ali, "On the Sombor index of graphs," Contributions to Mathematics, vol. 3, pp. 11-18, 2021.

[27] T. Došlic, T. Réti, and A. Ali, "On the structure of graphs with integer Sombor indices,” Discrete Mathematics Letters, vol. 7, pp. 1-4, 2021. 